\title{
Formulation of a Mathematical Model for the Analysis of the Emission of Carbon Dioxide from Gaseous Fuel using Least Square Method
}

\author{
Department of Mathematics, \\ Federal University of Technology, Minna, Nigeria \\ E-mail: m.shehu@futminna.edu.ng
}

${ }^{* 1}$ COLE, AT; ${ }^{2}$ SHEHU, MD; ${ }^{3}$ ABDULLAHI, A; ${ }^{4}$ BOLARIN, G

\begin{abstract}
In this paper we formulate a model for the emission of Carbon Dioxide from gaseous fuel in Nigeria. We considered a third degree polynomial model using a least square method using the actual data set of twenty one years on State level Carbon Dioxide emissions in Nigeria from 1994-2014. The residual analysis was used to predict the short and medium term total Carbon Dioxide emissions trend. From the results the state of affairs of Carbon Dioxide Emission for subsequent years was forecast and this will help the Government to take control measures in curtailing the emission of Carbon Dioxide in the country. (C) JASEM

https://dx.doi.org/10.4314/jasem.v21i5.4
\end{abstract}

.Keywords: Gaseous fuel, Automobile, Fossil fuels, Pollutants, Carbon Dioxide, Emissions

Research conducted globally reveals that the common atmospheric pollutant is carbon monoxide with a yearly percentage of $70 \%$ of all carbon monoxide gas emitted by automobiles (Ahmad, Noor, et al, 2014).

In every day's life a normal human being have an intake of approximately 13,000-16,000 litres of air and 400- 500 million litres of air (Bultije, 2014). The consequence of atmospheric pollutants to life differs in relation to vulnerability, quantity of atmospheric pollutants and also the living standard of the people. People without health challenges are not spare, especially, those that are prone to disease (Marion, 2009; Bellasio, 2007; Han- Ulrich, 2014).

The following are the effect of atmospheric pollutants to life; Raising cases lung cancer, High rate of chronic asthma, High rate of coughing/phlegm, High cases of upper respiratory system, High level of irritation cases in throat and other sensory organs (Oztirk, 2015).

Mahmmud (2012) carried out a comparative study on Air pollution and transportation in urban area. $\mathrm{He}$ stressed that has proven to be connected with how motor vehicles operate in the cities.

The aim of this paper is to formulate a mathematical model for the analysis of the emission of carbon dioxide from gaseous fuel using least square method

\section{MATERIALS AND METHODS}

We considered a third degree polynomial model using a least square method for gaseous fuels $(G)$.

We consider the equation,

$$
y(x)=a+b x+c x^{2}+d x^{3}
$$

where,

$y=$ total emission from gaseous fuel

$x=$ year

We define the error term as;

$$
E(a, b, c, d)=\sum_{n=1}^{N}\left\{y_{n}-\left(a+b x_{n}+c x_{n}^{2}+d x_{n}^{3}\right)\right\}^{2}
$$

Where our data set variables is defined as;

$$
E(a, b, c, d)=\left\{y_{1}-\left(a+b x_{1}+c x_{1}^{2}+d x_{1}^{3}\right)\right\}, \ldots,\left\{y_{N}-\left(a+b x_{N}+c x_{N}^{2}+d x_{N}^{3}\right)\right\}
$$

To minimizes the error, we find $\mathrm{a}$ and $\mathrm{b}$ this makes us find the values of $(a, b, c, d)$ thus;

$$
\frac{\partial E}{\partial a}=\frac{\partial E}{\partial b}=\frac{\partial E}{\partial c}=\frac{\partial E}{\partial d}=0
$$

Differentiating

$E(a, b, c, d)$ 
Partially we have;

$$
\begin{aligned}
& \frac{\partial E}{\partial a}=-2 \sum_{n=1}^{N}\left(y_{n}-a-b x_{n}-c x_{n}^{2}-d x_{n}^{3}\right) \\
& \frac{\partial E}{\partial b}=-2 \sum_{n=1}^{N} x_{n}\left(y_{n}-a-b x_{n}-c x_{n}^{2}-d x_{n}^{3}\right) \\
& \frac{\partial E}{\partial c}=-2 \sum_{n=1}^{N} x_{n}^{2}\left(y_{n}-a-b x_{n}-c x_{n}^{2}-d x_{n}^{3}\right) \\
& \frac{\partial E}{\partial d}=-2 \sum_{n=1}^{N} x_{n}^{3}\left(y_{n}-a-b x_{n}-c x_{n}^{2}-d x_{n}^{3}\right)
\end{aligned}
$$

Dividing by 2 we have,

$$
\begin{aligned}
& \sum_{n=1}^{N}\left(y_{n}-a-b x_{n}-c x_{n}^{2}-d x_{n}^{3}\right)=0 \\
& \sum_{n=1}^{N} x_{n}\left(y_{n}-a-b x_{n}-c x_{n}^{2}-d x_{n}^{3}\right)=0 \\
& \sum_{n=1}^{N} x_{n}^{2}\left(y_{n}-a-b x_{n}-c x_{n}^{2}-d x_{n}^{3}\right)=0 \\
& \sum_{n=1}^{N} x_{n}^{3}\left(y_{n}-a-b x_{n}-c x_{n}^{2}-d x_{n}^{3}\right)=0
\end{aligned}
$$

Representing (9), (10), (11), (12) in matrix form we have

$$
\left[\begin{array}{cccc}
\sum_{n=1}^{N} 1 & \sum_{n=1}^{N} x_{n} & \sum_{n=1}^{N} x_{n}^{2} & \sum_{n=1}^{N} x_{n}^{3} \\
\sum_{n=1}^{N} x_{n} & \sum_{n=1}^{N} x_{n}^{2} & \sum_{n=1}^{N} x_{n}^{3} & \sum_{n=1}^{N} x_{n}^{4} \\
\sum_{n=1}^{N} x_{n}^{2} & \sum_{n=1}^{N} x_{n}^{3} & \sum_{n=1}^{N} x_{n}^{4} & \sum_{n=1}^{N} x_{n}^{5} \\
\sum_{n=1}^{N} x_{n}^{3} & \sum_{n=1}^{N} x_{n}^{4} & \sum_{n=1}^{N} x_{n}^{5} & \sum_{n=1}^{N} x_{n}^{6}
\end{array}\right]\left[\begin{array}{l}
a \\
b \\
c \\
d
\end{array}\right]=\left[\begin{array}{c}
\sum_{n=1}^{N} y_{n} \\
\sum_{n=1}^{N} x_{n} y_{n} \\
\sum_{n=1}^{N} x_{n}^{2} y_{n} \\
\sum_{n=1}^{N} x_{n}^{3} y_{n}
\end{array}\right]
$$

The matrice is in general invertible and it implies the estimated values of a, b, c and d. Thus,

$$
\left[\begin{array}{l}
a \\
b \\
c \\
d
\end{array}\right]=\left[\begin{array}{cccc}
\sum_{n=1}^{N} 1 & \sum_{n=1}^{N} X_{n} & \sum_{n=1}^{N} x_{n}^{2} & \sum_{n=1}^{N} x_{n}^{3} \\
\sum_{n=1}^{N} x_{n} & \sum_{n=1}^{N} x_{n}^{2} & \sum_{n=1}^{N} x_{n}^{3} & \sum_{n=1}^{N} x_{n}^{4} \\
\sum_{n=1}^{N} x_{n}^{2} & \sum_{n=1}^{N} x_{n}^{3} & \sum_{n=1}^{N} x_{n}^{4} & \sum_{n=1}^{N} x_{n}^{5} \\
\sum_{n=1}^{N} x_{n}^{3} & \sum_{n=1}^{N} x_{n}^{4} & \sum_{n=1}^{N} x_{n}^{5} & \sum_{n=1}^{N} x_{n}^{6}
\end{array}\right]^{-1}\left[\begin{array}{l}
\sum_{n=1}^{N} y_{n} \\
\sum_{n=1}^{N} x_{n} y_{n} \\
\sum_{n=1}^{N} x_{n}^{2} y_{n} \\
\sum_{n=1}^{N} x_{n}^{3} y_{n}
\end{array}\right]
$$


Data Presentations: For formulation and analysis of our model, the following emission data from 1994 to 2014 is used..

Table 1: Real data of emission from gaseous fuel in Nigeria ('000 MT of carbon dioxide)

\begin{tabular}{ll}
\hline Year & Gas \\
\hline 1994 & 4.24 \\
1995 & 5.25 \\
1996 & 7.22 \\
1997 & 7.59 \\
1998 & 9.29 \\
1999 & 12.59 \\
2000 & 18.84 \\
2001 & 21.17 \\
2002 & 26.80 \\
2003 & 30.04 \\
2004 & 41.46 \\
2005 & 39.34 \\
2006 & 36.12 \\
2007 & 30.61 \\
2008 & 43.11 \\
2009 & 50.50 \\
2010 & 63.98 \\
2011 & 37.86 \\
2012 & 41.54 \\
2013 & 50.61 \\
2014 & 45.52 \\
\hline
\end{tabular}

Source: Hans-Ulrich (2014) State level gaseous emissions of Nigeria (1994-2014).

\section{RESULTS AND DISCUSSION}

Gaseous fuels: The general mathematical model for the emission of gaseous fuel is given by:

$$
y(x)=a+b x+c x^{2}+d x^{3}(15)
$$

where, $\mathrm{x}=$ year

The solution of the above equation is given by
Table 2: Values of $\mathrm{R}^{2}$ and $\mathrm{R}^{2}$ adjusted on gaseous fuel

\begin{tabular}{cc}
\hline$R^{2}$ & $R_{\text {adjusted }}^{2}$ \\
\hline $\mathbf{0 . 8 3 1 6}$ & 0.8018 \\
\hline
\end{tabular}

Table 3: Residual analysis for emissions from gaseous fuels ('000 MT of carbon dioxide)

\begin{tabular}{llll}
\hline Year & $\begin{array}{l}\text { Real Data } \\
\text { (See Table 4.1) }\end{array}$ & $\begin{array}{l}\text { Generated for } \\
\text { Model Data }\end{array}$ & Residual \\
\hline 1994 & 4.24 & 4.12 & 0.12 \\
1995 & 5.25 & 6.66 & -1.41 \\
1996 & 7.22 & 9.20 & -1.97 \\
1997 & 7.59 & 11.75 & -4.15 \\
1998 & 9.29 & 14.30 & -5.00 \\
1999 & 12.59 & 16.85 & -4.26 \\
2000 & 18.84 & 19.41 & -0.56 \\
2001 & 21.17 & 21.97 & -0.26 \\
2002 & 26.8 & 24.53 & 2.27 \\
2003 & 30.04 & 27.10 & 2.94 \\
2004 & 41.46 & 29.67 & 11.79 \\
2005 & 39.34 & 32,24 & 7.10 \\
2006 & 36.12 & 34.81 & 1.30 \\
2007 & 30.61 & 37.39 & -6.78 \\
2008 & 43.11 & 39.98 & 3.13 \\
2009 & 50.50 & 42.56 & 7.93 \\
2010 & 63.98 & 45.15 & 18.83 \\
2011 & 37.86 & 47.74 & -9.88 \\
2012 & 41.54 & 50.34 & -8.79 \\
2013 & 50.61 & 52.94 & -2.32 \\
2014 & 45.52 & 55.54 & -10.01 \\
Mean of Residuals & & & $8.07 \mathrm{E}-$ \\
& & & 0.005 \\
Standard error of & & & 2.67 \\
Residuals (SE) & & & \\
\hline
\end{tabular}

Table 4: Emission from gaseous fuels ('000 MT of carbon dioxide)

\begin{tabular}{ll}
\hline Year & Gas Emission \\
\hline 2015 & 72.29 \\
2016 & 83.67 \\
2017 & 95.06 \\
2018 & 96.44 \\
2019 & 97.84 \\
2020 & 99.23 \\
$x^{3} 2021$ & 100.62 \\
2022 & 102.02 \\
2023 & 103.42 \\
2024 & 112.29 \\
\hline
\end{tabular}

(16)

The result of $\mathrm{R}^{2}$ and $\mathrm{R}^{2}$ adjusted on gaseous fuel model are shown in the Table below.

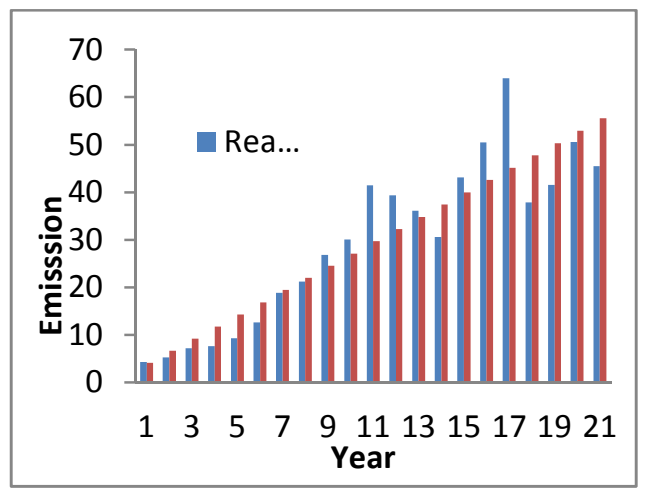

Fig 1: Emission from gaseous fuels ('000 MT of carbon dioxide)

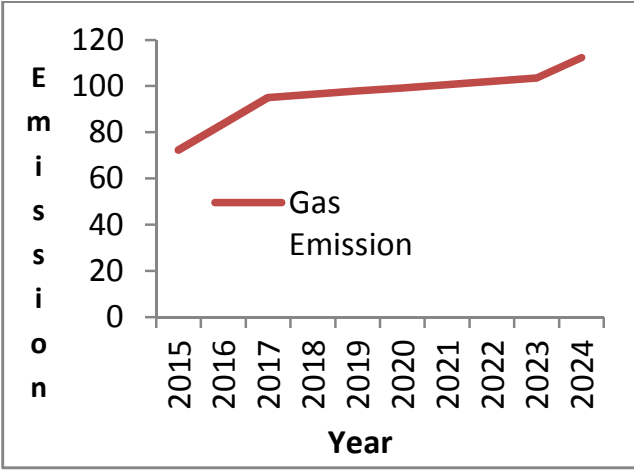

Fig 2: Emission of $\mathrm{CO}_{2}$ from Gaseous fuels ('000 MT of carbon dioxide)

Figure 2 shows the emission from Gaseous fuels for short and medium term. From the study, we can say 
that appropriate measures are immediately required to control the rapid increasing of gas emission in Nigeria. Our prediction may be helpful for anticipated planning and prolific policies to curb the emission rate of carbon dioxide from different sources. We therefore recommend that government should use free carbon dioxide emission sources of energy such as; solar power, wind power, geothermal energy, low-head hydropower, hydrokinetics (wave and tidal power) and nuclear power

\section{REFFERENCES}

Ahmad Z; Noor B, Sharifa A. (20014), Effect of carbon dioxide emissions in Nigeria, IOSR Journal of Applied Chemistry 1: 06-10.

Bellasio R (2007), Emission of carbon dioxide from different attributes in India: A Mathematical study, IOSR Journal of Applied Chemistry 1: 1023.

Bultije C (2014), The Carbon cycle and atmospheric carbon dioxide. Journal of Nonlinear Science. 5(6): 180-185.
Han- Ulrich (2014), The Dynamic evolutionary analysis on carbon emissions in Yangtze delta, International. Journal of Nonlinear Science 10(3): 259-263.

Mahmmud K (2014), Evaluation of Air Pollution Trends in İstanbul. International Journal of Environment and Pollution 18(4):388-398.

Marion C P. (2009). Modeling carbon dioxide emissions with a system of differential equations, NonlinearAnalysis, International Journal of Science and Technology 3(3): $184-187$.

Oztirk A. (2015). Global warming and infectious disease. Journal of advances in Environmental Research 3(1), 45 - 69 